\title{
Composants à fibres optiques
}

\author{
J.P. Goure
}

Laboratoire Traitement du Signal et Instrumentation, Unité Associée au CNRS 842, Rue du Docteur Paul Michelon, F-42023 Saint-Etienne cedex, France

\section{1- INTRODUCTION}

Le développement rapide de la technologie fibre optique dans les télécommunications et plus récemment en instrumentation a nécessité un besoin croissant de composants actifs et passifs en optique guidée. Par exemple des composants optiques tels que les coupleurs source-fibre, les épissures, les connecteurs, les coupleurs directionnels, les multiplexeurs, les commutateurs et modulateurs sont des éléments essentiels pour les transmissions et les capteurs. Le but de cet article est de donner une idée générale des possibilités des composants à fibre et de leur réalisation. Le lecteur pourra trouver des explications plus complètes et plus détaillées dans des articles et livres spécialisés [1-16]. Dans le tableau I les principaux composants à fibre et leur principes physiques sont listés.Les composants en optique intégrée qui sont réalisés en surface de substrats plans ne sont pas traités dans cet exposé, bien qu'ils présentent un intérêt évident. Ils nécessitent à eux seuls des développements[17-18].

\section{2 - EXPANSEUR DE FAISCEAU}

L'utilisation de fibres optiques monomodes exige l'emploi de composants fiables. La fabrication de ces composants est difficile, à cause de la très faible dimension du coeur. Les composants associés aux guides monomodes sont très sensibles aux déplacements axiaux et transversaux de nature mécanique ou thermiques, ainsi qu'aux petites particules de poussières. L'introduction d'éléments optiques élargisseurs de faisceaux peut éviter ces problèmes. Le rôle essentiel d'un "taper" est de dilater la faisceau monomode (Figure 1). Les "tapers" coniques sont des portions de fibres dont le diamètre

change continument le long de leur axe. Ils peuvent être réalisés très simplement en chauffant une partie de fibre à l'aide d'un chalumeau et en appliquant une contrainte axiale. En coupant la partie biconique à l'endroit de son rétrécissement, deux "tapers" à fibres coniques sont alors obtenus. Dans un composant qui se retrécit le rayon de coeur et la fréquence normalisée diminuent tandis que la dimension du spot (spot size) croit. Le faisceau quand il se propage dans un milieu homogène ne peut être rétréci très vite à cause de la diffraction. Ainsi dans un "taper" qui concentre fortement la lumière, beaucoup d'énergie guidée est convertie en énergie radiée.

Ces systèmes peuvent avoir une géométrie monomode standard à une extrémité et augmenter progressivement leur section transverse de telle manière à ce que la taille du coeur à l'autre extrémité soit comparable à celle d'une fibre multimode. Pour un "taper" avec une évolution géomètrique suffisamment lente, les fronts d'ondes restent pratiquement plans. Il a été montré que pour un "taper" en 


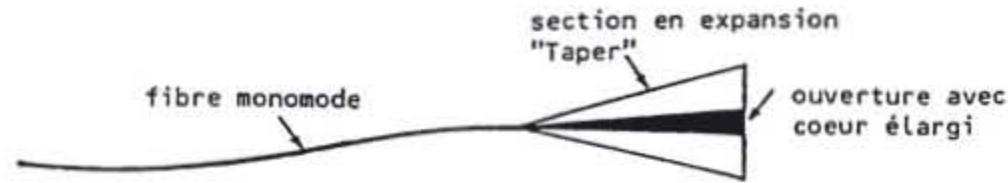

(a)

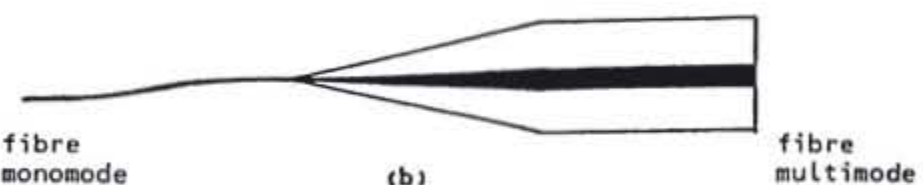

Figure 1
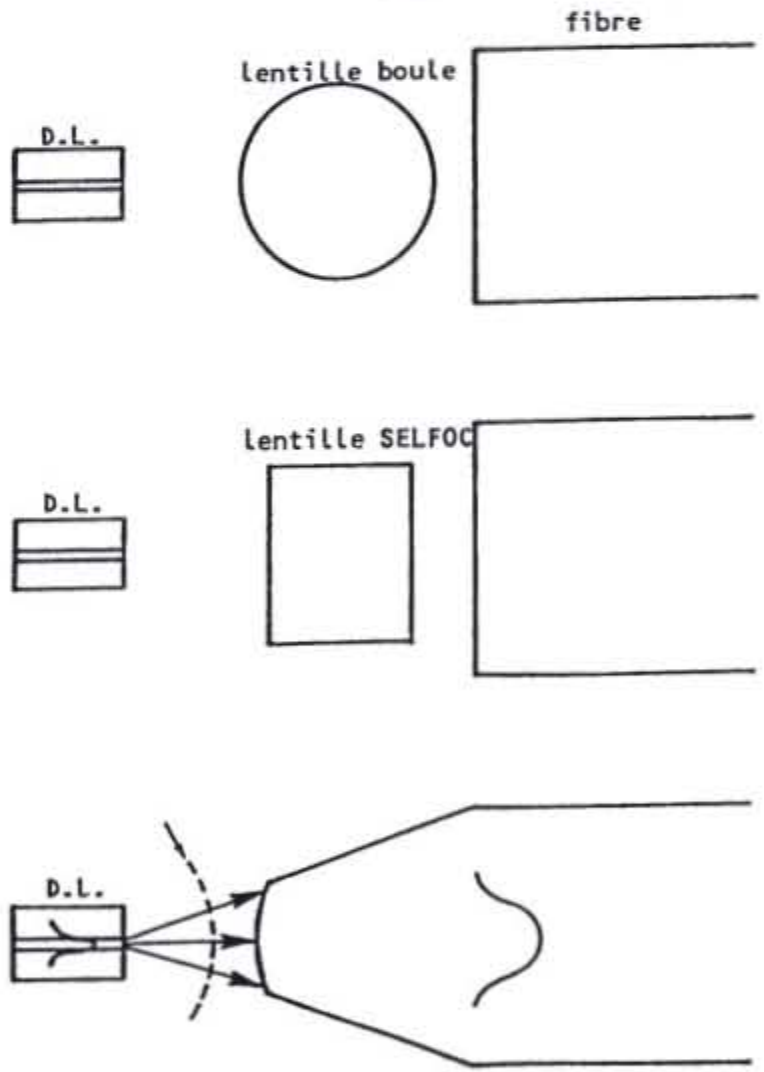

Figure 2 
"pente douce", qui à son extrémité large peut propager des ondes de manière multimode, la conversion du mode fondamental en d'autres modes guidés d'ordres plus élevés peut être négligeable si un faible . taux de pertes

de couplage en exercés est toléré. Ces composants peuvent être couplés à d'autres composants avec très peu de pertes (inférieur à $0,1 \mathrm{~dB}$ ).

De plus, dans les modules de transmissions, capteurs ou instruments, le maximum d'efficacité de couplage optique entre laser à semi conducteur et fibre monomode ainsi que le minimum de réflexion optique parasite dans le laser sont exigés. Beaucoup de systèmes de couplage optiques ont été envisagés. Des composants tels que lentilles, lentilles à gradient d'indice (GRIN lenses), microlentilles sphériques ou "taper"s sont utilisés . Le "taper" à fibre étirée avec une microlentille sphérique intégrée à sont extrémité à une efficacité de couplage supérieure à $55 \%$ avec une lentille de rayon d'environ 10 $\mu \mathrm{m}$. Le "taper" à fibre et lentille en quartz assure des efficacités de couplage entre 45 et $50 \%$ avec une bonne reproductibilité.

\section{3 - COUPLEURS DIRECTIONNELS}

\section{3 - 1 Coupleurs en X, en Y, coupleurs "étoile"}

Les composants à base de fibres pour diviser ou additionner des signaux, et appelés coupleurs de puissance, sont les plus utilisés dans la distributions de données, dans les systèmes multiplexeurs démultiplexeurs, dans les systèmes de transmission cohérente, dans les capteurs à fibres et l'instrumentation associée.

Un coupleur est un composant passif qui distribue la puissance d'une fibre principale vers une ou plusieurs autres fibres. Ils peuvent être classés en coupleurs indépendants de la polarisation et en coupleurs dépendants de la polarisation. Ces derniers sont à leur tour classés en coupleurs à maintien de polarisation ou en coupleur à séparation de polarisation. La figure 3 montre un coupleur 2 par 2.

Dans un coupleur en X l'énergie est transférée d'une fibre optique multimode excitée à une fibre multimode parallèle adjacente du fait qu'une part appréciable de l'énergie est propagée dans la gaine par l'onde évanescente. Pour des fibres monomodes, ce sont les deux modes fondamentaux qui sont couplés à travers leur champ évanescent . Les coeurs doivent être aussi proches que possible. Les rapports des diamètres coeur/gaine et la séparation entre les coeurs ont des effets significatifs sur les caractéristiques.

Plusieurs techniques ont été mises au point pour réaliser ces coupleurs et différentes sortes ont été proposées utilisant des fibres et des composants en micro-optique : coupleurs à gaine polie, coupleurs biconiques obtenus par fusion étirage, séparateurs de faisceaux...

Une méthode générale pour les fabriquer consiste à polir un côté du coeur des deux fibres et à les amener à proximité avec un liquide adaptateur d'indice (Figure 4). Les fibres sont fixées dans des rainures pratiquées dans des blocs de quartz. Les blocs sont polis sur un côté de manière à éroder une partie de la gaine. La puissance transférée dépend de la longueur d'interaction. 
Une autre méthode de fabrication permet de réaliser des coupleurs avec deux fibres ou plus (coupleur étoile). Cette méthode consiste à attaquer chimiquement la gaine de la fibre avec une solution d'acide

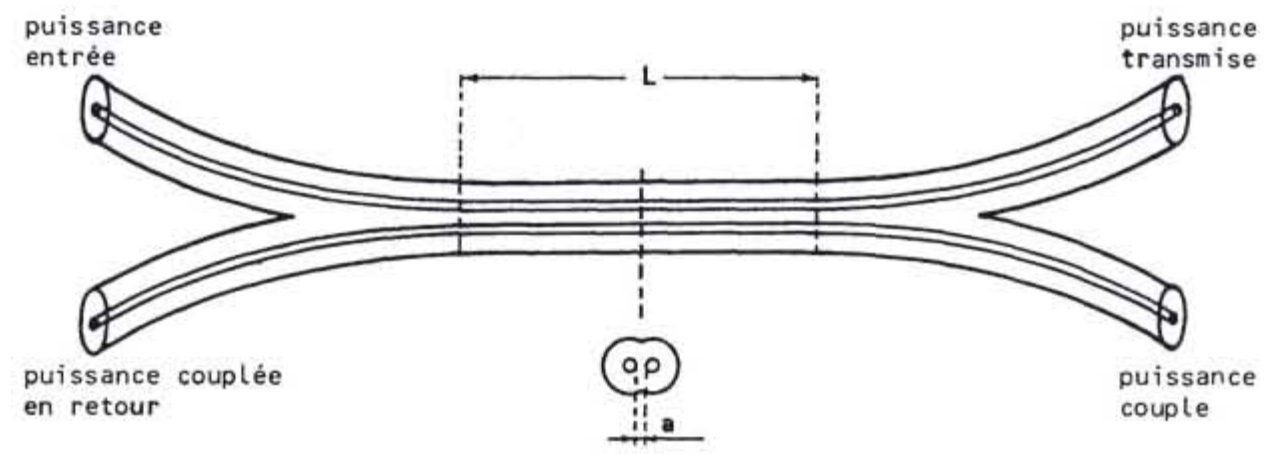

Figure 3

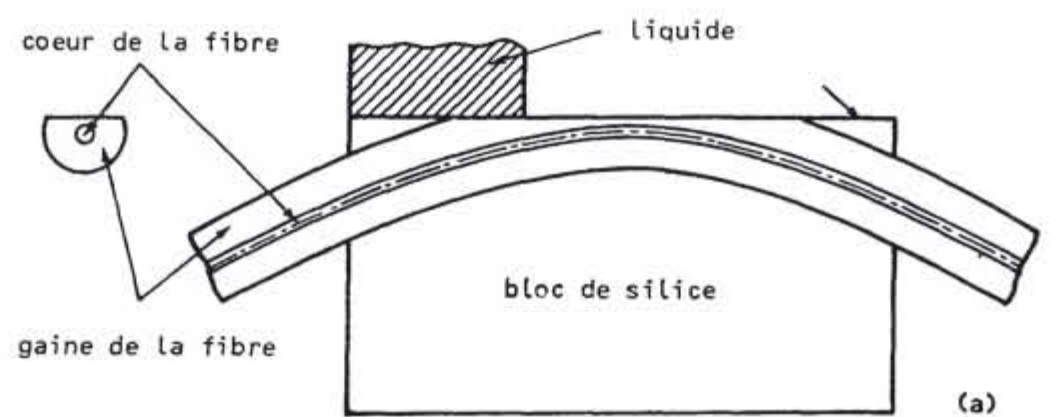

(a)

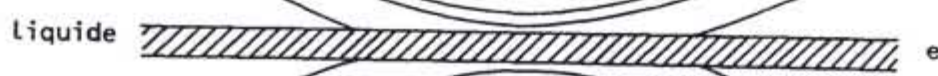

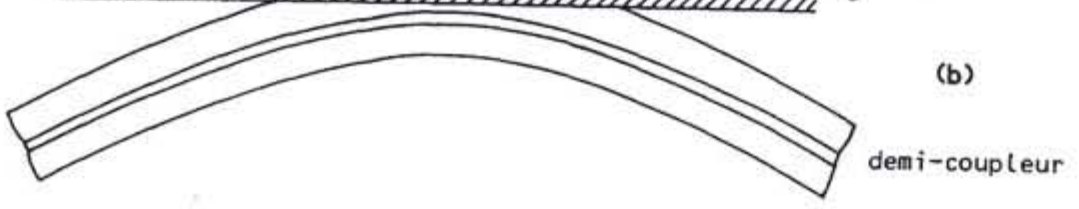

Figure 4 
fluorhydrique, puis de les entourer les unes sur les autres. Elles sont ensuite fondues à la flamme et étirées. Ces composants ont des faibles pertes $(0,5 \mathrm{~dB})$ (Figure 5).Certains coupleurs ont été fabriqués avec des fibres différentes en vue d'applications données par exemple dans le cas de lasers à fibre. Les coupleurs $2 \times 2$ monomodes ont une réponse en longueur d'onde plate et sont utilisés dans des applications demandant un rapport de séparation presque constant sur un large domaine spectral.

Le principe est basé sur les équations de couplage des ondes. Considérons deux guides parallèles quand une puissance unité est injectée dans l'une des fibres et qu'aucune puissance ne l'est dans la seconde les puissances reçues en sortie après un parcours $\mathrm{z}$ sont :

$$
\begin{aligned}
& P_{1}(z)=1-F^{2} \sin ^{2} \frac{C}{F} z \\
& P_{2}(z)=F^{2} \sin ^{2} \frac{C}{F} z
\end{aligned}
$$

C est le coefficient de couplage. Une fraction $\mathrm{F}^{2}$ de la puissance est transférée d'une fibre à l'autre et revient sur la première après une longueur de battement : $L=\frac{2 \pi F}{C}$

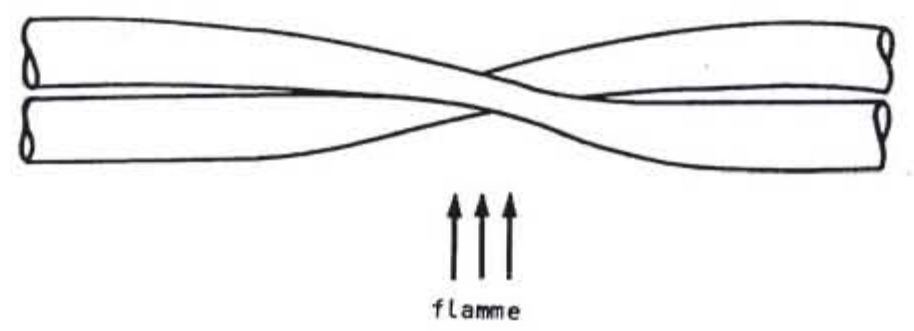

Figure 5

Dans le cas simple de deux fibres identiques $(\mathrm{F}=1)$ et dans l'approximation de guidage faible, on obtient pour un coupleur biconique obtenu par fusion à partir de fibres monomodes

$$
\mathrm{C}(\mathrm{z})=1.018 \frac{\lambda^{5 / 2}}{\left[\mathrm{n}_{2}^{2}-\mathrm{n}_{3}^{2}\right]^{3 / 4} \mathrm{n}_{2}[\mathrm{~b}(\mathrm{z})]^{3 / 2}}
$$

et un rapport de couplage entre les deux fibres: $C R=P_{2} /\left(P_{1}+P_{2}\right)$

$\mathrm{n}_{2}$ est l'indice de gaine, $\mathrm{n}_{3}$ l'indice du milieu. Les deux coeurs disparaissent à l'endroit de la fusion [Figure 6]. 
Une autre catégorie de coupleur utilise les composants en micro-optique : lentilles selfoc, miroir, réseaux (Figure 7). Le coupleur en $\mathrm{Y}$ est semblable au coupleur en $\mathrm{X}$ et réalisé suivant les mêmes techniques (Figure 8) : gaine polie, fusion étirage ... Un coupleur en étoile est utilisé pour distribuer l'énergie sur un grand nombre de terminaux. Plusieurs techniques permettent de les réaliser ; l'une d'entre elles consiste à se servir de coupleur $2 \times 2$ (Figure 9).

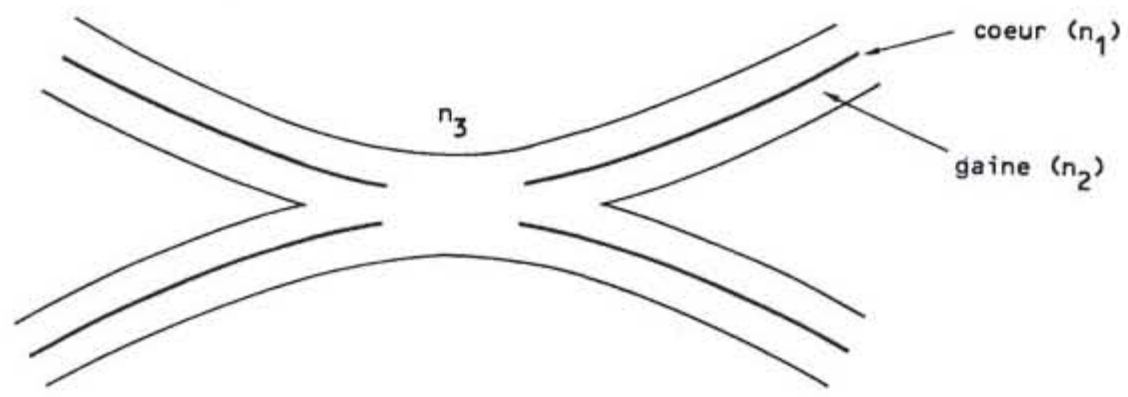

Figure 6

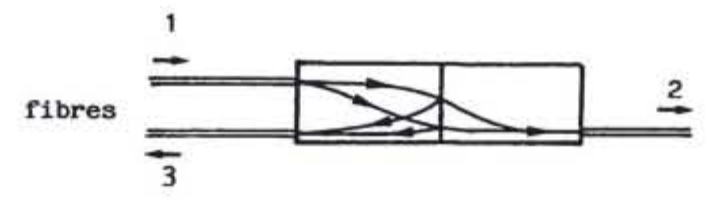

(a)
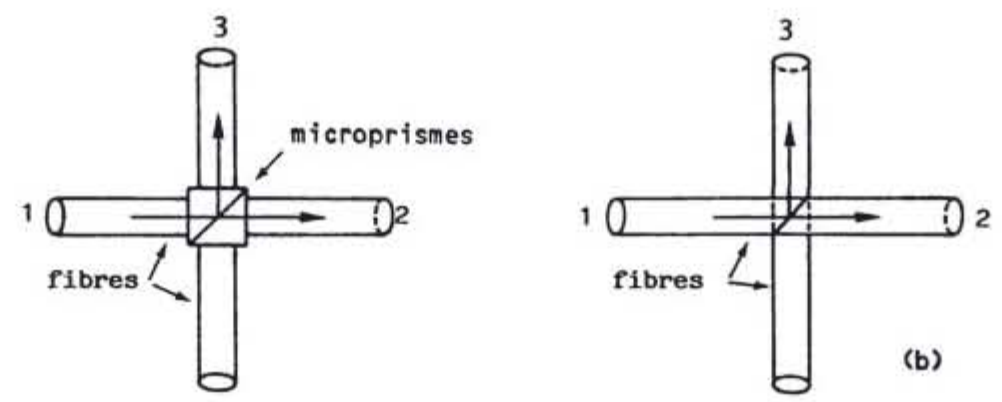

Figure 7.a.b

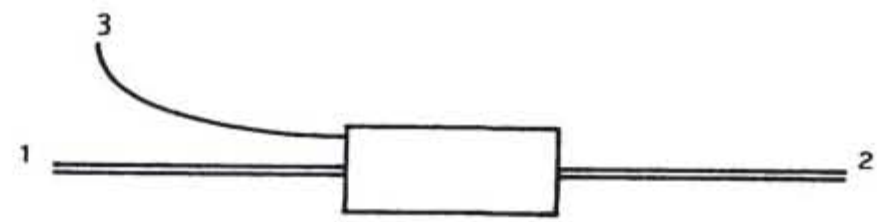

Figure 8 
(a)

$$
\text { I }
$$
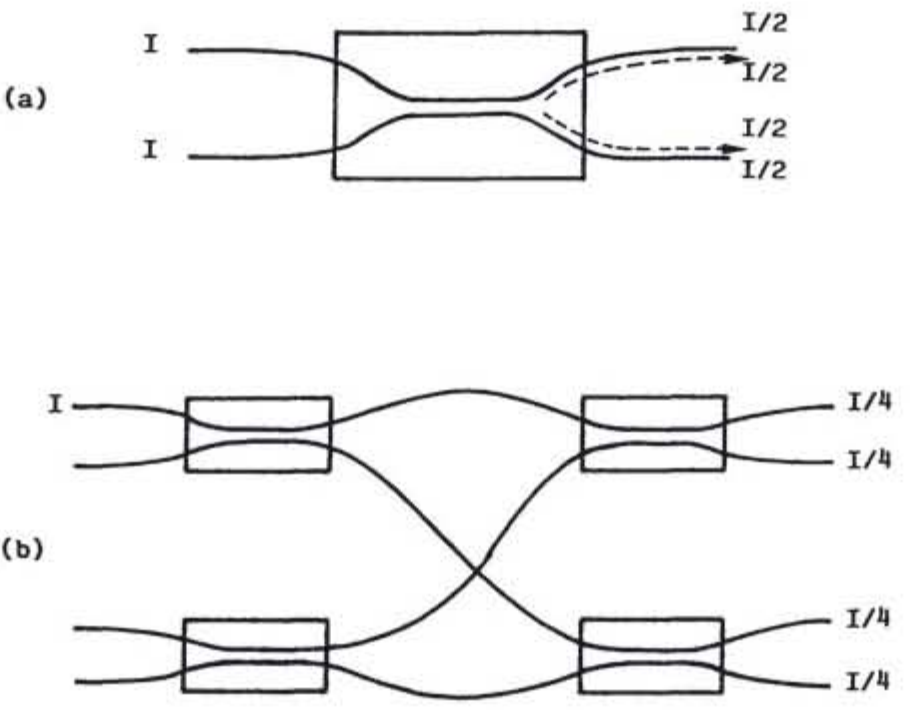

Figure 9

\section{3-2 Coupleur à maintien de polarisation et séparateur de polarisation}

Ces composants sont fabriqués à l'aide de fibres à maintien de polarisation. Les axes principaux des fibres doivent être parallèles et alignés avec précision. La figure 10a donne la structure de ces composants réalisés avec des fibres PANDA. Ces fibres présentent des zones avec un dopage différent des contraintes de part et d'autre du coeur. L'indice de réfraction y est plus bas que dans la gaine. Si la région de couplage est suffisamment longue, la dépendance de la polarisation devient importante. La polarisation $x$ est correctement couplée à l'autre coeur, la polarisation y est recouplée sur la pression fibre et une séparation de la polarisation est observée (Figure 10b).

\section{4 - FILTRES DE LONGUEURS D'ONDE}

Plusieurs sortes de filtres ont été réalisés avec des fibres monomodes. On peut par exemple courber légèrement une fibre sur un bloc dans une rainure et la coller. La surface du substrat est polie jusque près du coeur (Figure 11a). Un guide $\mathrm{n}_{\mathrm{e}}$ est placé sur la fibre dont la gaine a été enlevée pour être exposée au champ évanescent. Le mode fondamental se propage dans des conditions idéales sans perte 
avec une constante $B$ et un indice effectif $n_{e f}=B / k(k=2 \pi / \lambda)$; mais $B$ et $n_{e f}$ dépendent de la longueur d'onde $\lambda$. Si une partie de la gaine a un indice $n_{e}$ plus grand que $n_{e f}$ il agit comme une pompe pour $\lambda$. La perte augmente si $\lambda$ augmente parce que le champ du mode s'étend dans la gaine et que la différence $\mathrm{n}_{\mathrm{e}}-\mathrm{n}_{\mathrm{ef}}$ augmente.

(a)<smiles>CC(C)C</smiles>
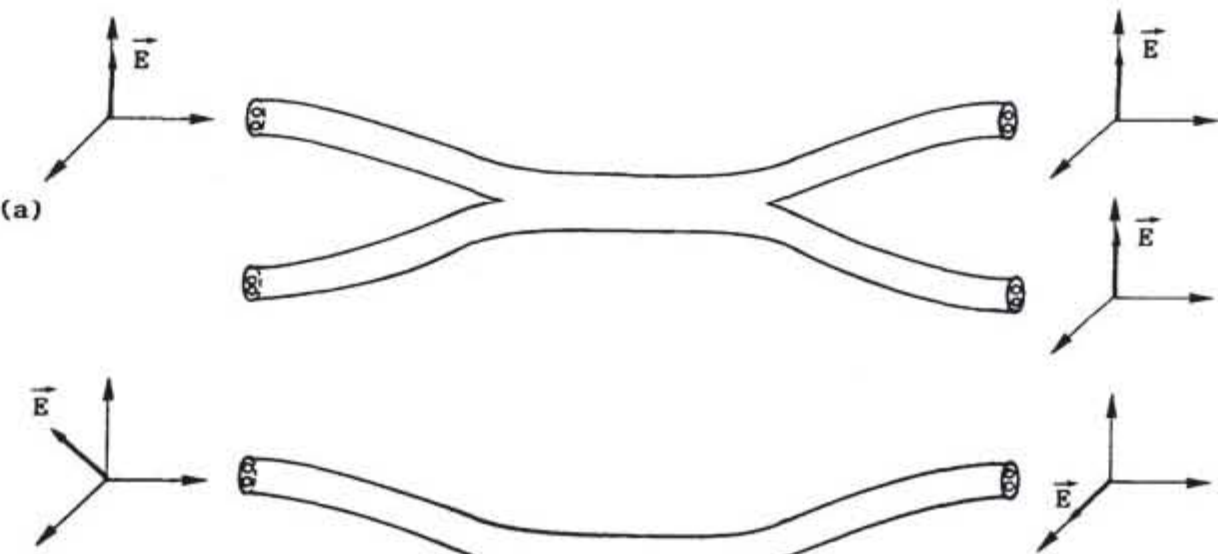

(b)
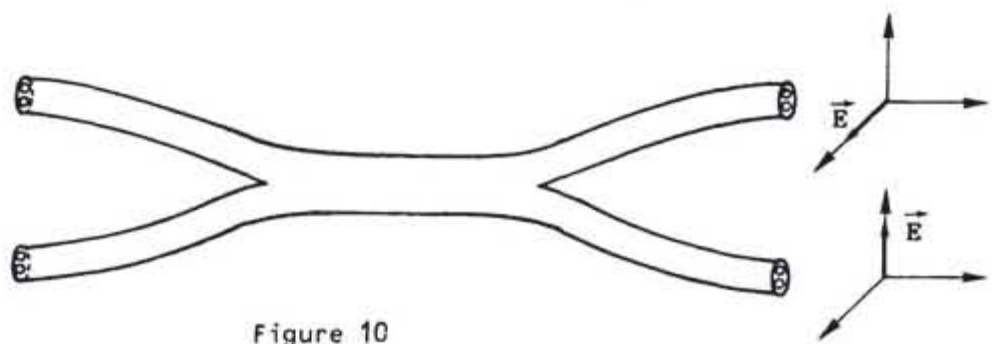

Dans un second montage le guide d'onde est recouvert par une couche de silice dont l'indice $\mathrm{n}_{\mathrm{S}}$ est égal à celui $\mathrm{n}_{\mathrm{cl}}$ de la gaine. pour un guidage directionnel efficace les conditions d'accord de vitesse de phase nécessitent que l'indice du film liquide et l'indice effectif du mode de la fibre soient égaux $\left(\mathrm{n}_{\mathrm{el}}=\mathrm{n}_{\mathrm{ef}}\right)$. Guidage et couplage ont lieu quand $n_{1}>n_{e f}>n_{s}$ (Figure 11b). La longueur d'onde $\lambda_{m}$ pour laquelle le mode de la fibre est accordé au mode du film est

$$
\lambda_{\mathrm{m}}=\frac{2 \mathrm{~d}}{\mathrm{~m}}\left(\mathrm{n}_{\mathrm{l}}^{2}-\mathrm{n}_{\mathrm{ef}}^{2}\right)^{1 / 2}
$$

où $\mathrm{m}$ est l'ordre du mode propagé à $\lambda_{\mathrm{m}}$ dans le guide plan d'épaisseur $\mathrm{d}$, dans la direction de l'axe de la fibre. Si la longueur d'interaction du couplage fibre / plan est égale à la longueur de couplage, la lumière à la longueur d'onde $\lambda_{\mathrm{m}}$ sort de la fibre dans le guide. Deux composants peuvent être réalisés : l'un avec un guide peu épais qui donne une réponse à saut (Figure 12a), le second avec un guide plus épais donne une réponse en peigne (Figure 12b) (raies séparées de $13 \mathrm{~nm}$ ). Si l'on utilise une fibre étirée de manière à réaliser un réducteur - expanseur (Figure 13a), la puissance peut être transférée du coeur à la gaine. Un coupleur coaxial apparait comme une suite de coupleurs si l'épaisseur est au dessous de la valeur définie par l'épaisseur d'accord. En ajustant au mieux le processus de fabrication, la réponse en longueur d'onde peut apparaître sinusoïdale :

$$
P_{q}(\lambda)=\frac{1}{2}\left[\left(1+\sin \left(\frac{2 \pi}{\delta \lambda}\left(\lambda-\lambda_{q}\right)\right)\right]\right.
$$



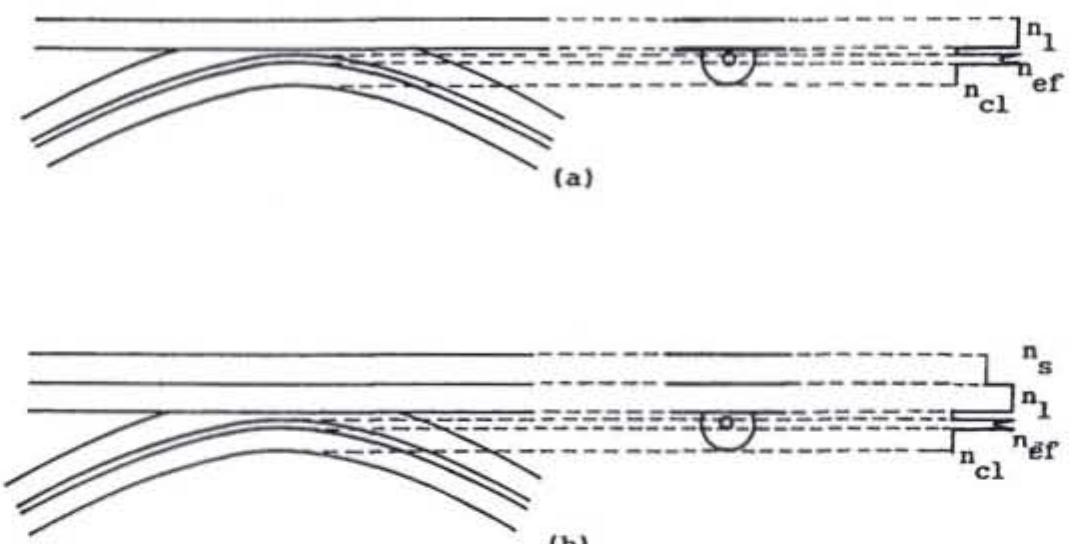

(b)

Figure 11

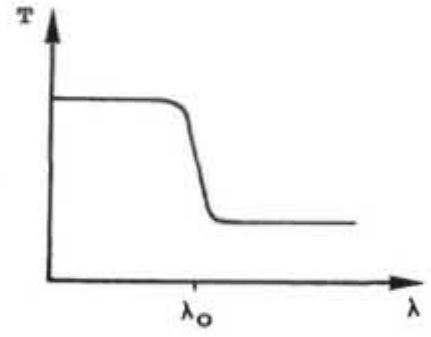

(a)

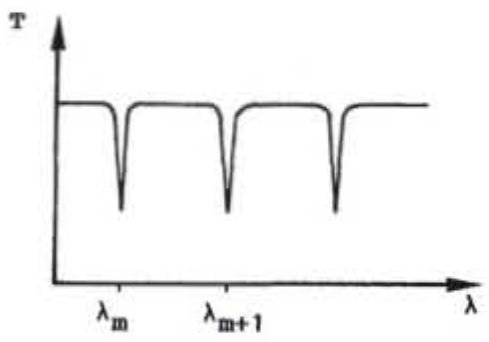

(b)

Figure 12

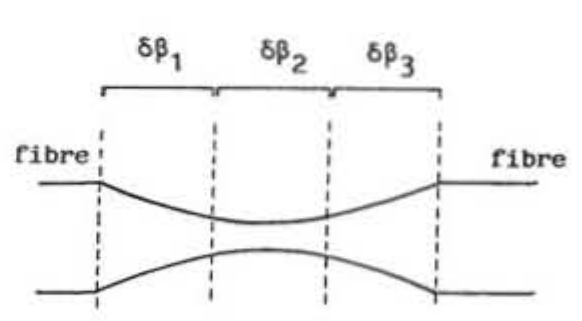

(a)

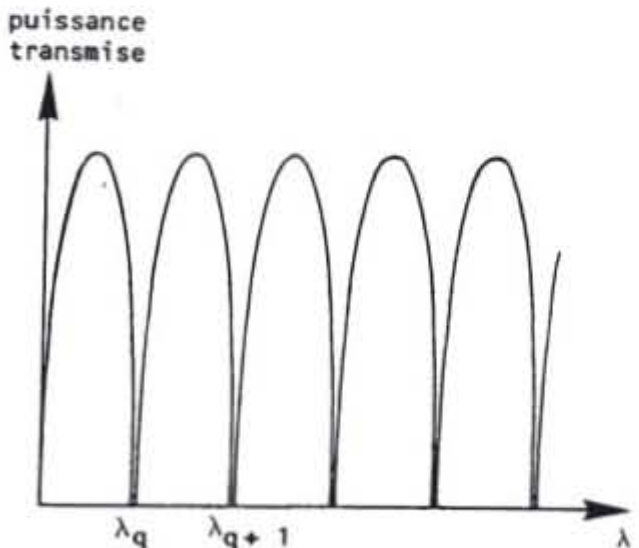

(b)

Figure 13 
$\delta \lambda$ est la période de la réponse du filtre (Figure 13b).

D'autres structures ont été proposées utilisant les réseaux gravés par attaque sur une fibre polie et recouverte avec une gaine à haut indice de réfraction (Figure 14). Ce composant a plus de $90 \%$ de réfléctivité dans le premier ordre du réseau de Bragg et est utilisé comme filtre, miroir, modulateur et commutateur.

Dans les applications capteurs ou en interférométrie, un couplage périodique entre les modes d'une même fibre peut être utilisé. Le couplage entre les modes $\mathrm{LP}_{01}$ et $\mathrm{LP}_{11}$ s'obtient en provoquant des micro courbures dans la fibre (Figure 15) ou en la serrant. Un coupleur basé sur des micro courbures dont la période est égale à la longueur de battement $\mathrm{L}_{\mathrm{B}}$ entre les modes peut être réalisé. Cette longueur $L_{B}$ est égale à $2 \pi / \Delta B$ ou $\Delta B$ est la différence entre les constantes de propagation des deux modes. Par exemple $\mathrm{L}_{\mathrm{B}}=270$ et $265 \mu \mathrm{m}$ à $\lambda=590$ et $496,5 \mathrm{~nm}$ pour une fibre de diamètre de coeur $2.28 \mu \mathrm{m}$ et de longueur d'onde de coupure à $671 \mathrm{~nm}$. Le couplage maximum obtenu est de 99,7\%.

\section{5 - DECALAGE EN LONGUEUR D'ONDE}

Dans une fibre rectiligne, les deux modes $\mathrm{LP}_{01}$ et $\mathrm{LP}_{11}$ sont orthogonaux et n'échangent pas d'énergie lorsqu'ils se propagent. On peut toutefois les coupler par des micro-courbures périodiques dont la période est adaptée à la longueur de battement. Ceci peut être obtenu par une onde acoustique.

Une fibre biréfringente conduit deux modes polarisés et du fait d'une différence entre leurs constantes de propagation, il y a un léger couplage entre ces modes. Si la fibre est comprimée périodiquement un transfert total de puissance peut être observé. L'utilisation d'une onde acoustique peut produire la compression périodique, et la lumière peut être couplée entre les deux principales polarisations de la fibre biréfringente. La condition d'accord de phase est $\Lambda=L_{B}$ où $\Lambda$ est la longueur d'onde acoustique et $\mathrm{L}_{\mathrm{B}}$ la longueur de battement $\mathrm{L}_{\mathrm{B}}=\lambda / \Delta \mathrm{n}$ où $\Delta \mathrm{n}$ est la biréfringence. La puissance couplée d'une polarisation à l'autre atteint alors un maximum. En modifiant la fréquence de l'onde acoustique la longueur d'onde centrale peut être modifiée. Un prototype réalisé avec une forme de section principale en $\mathrm{D}$ a permis de faire varier de 570 à $630 \mathrm{~nm}$ la longueur d'onde par une modification de la fréquence de 2.85 à $2.55 \mathrm{MHz}$.

\section{6 - POLARISEURS}

Ils sont réalisés en remplaçant la gaine par un cristal biréfringent ou un métal. Quand une lumière polarisée avec un spectre étroit est injectée dans une fibre monomode, un haut degré de polarisation est maintenu sur une grande longueur. Cet état de polarisation varie cependant suivant les contraintes extérieures, ce qui conduit à du bruit lorsque des polariseurs ou des réseaux sont introduits. Pour réduire ce type de bruit des dépolariseurs ont été réalisés. Un exemple est un dépolariseur de LYOT fait par deux fibres avec un support de longueur $2 / 1$ et leurs axes principaux à $45^{\circ}$ l'un de l'autre. Cest la différence de délai de groupe entre les deux modes principaux qui est utilisée. 


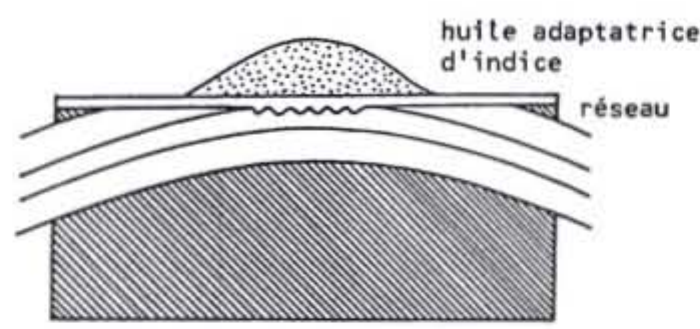

Figure 14

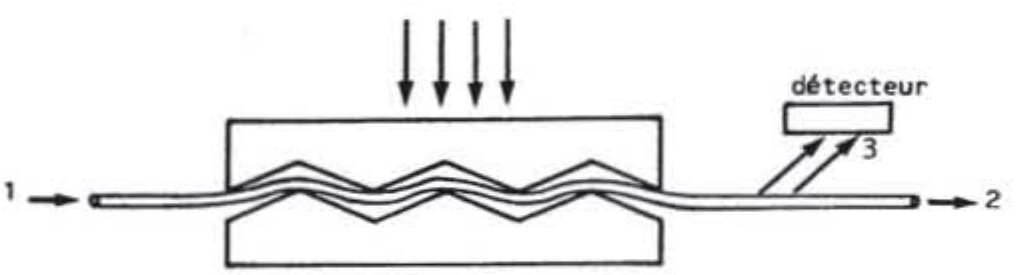

Figure 15

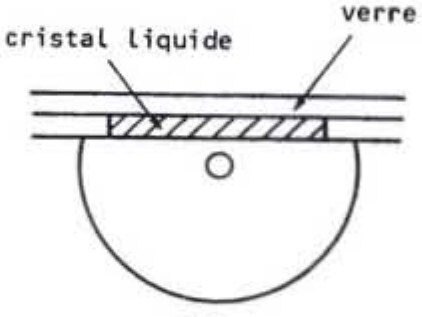

(a)

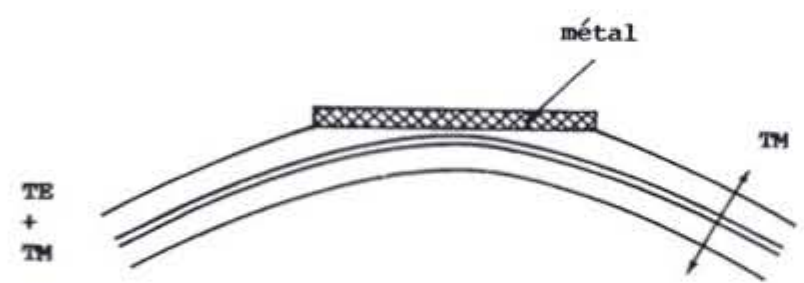

(b)

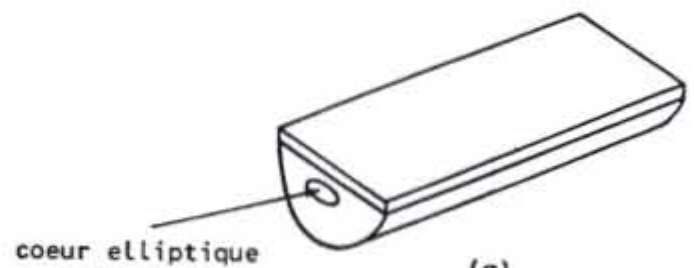

(c)

Figure 16 
Un polariseur à fibre peut être réalisé à l'aide d'un cristal biréfringent placé près du coeur. Le champ évanescent interagit avec le cristal obligeant la polarisation non désirée à se coupler hors de la fibre. Un cristal liquide nématique a permis une sélection de $45 \mathrm{~dB}$ avec une perte d'insertion de $1,1 \mathrm{~dB}$. On utilise un demi coupleur (Figure 16a). La variation d'amplitude s'effectue en contrôlant l'alignement des molécules du cristal avec un champ électrique. Quand l'alignement est produit, un état de polarisation verra l'indice élevé et ne sera pas guidé tandis que la polarisation orthogonale verra l'indice faible et sera guidée. Le cristal doit être chauffé entre 62 et $85^{\circ} \mathrm{C}$.

On peut aussi utiliser un film mince métallique. Le principe repose sur l'atténuation différentielle des deux polarisations ou sur le "cutt-off" du mode TEo. On réduit le diamètre de la fibre par polissage si bien que la fibre est en dessous de la condition de "cut off". On dépose une fine couche de métal sur la partie plane (Figure 16b). Seule l'onde TM satisfait la condition limite et se propage à l'interface. Cette lumière se convertit en plasmon de surface qui se propage dans la région d'interaction et se reconvertit en une polarisation TM dans la fibre. Le mode TE est perdu dans la gaine. Un taux d'extinction de 47 $\mathrm{dB}$ a été mesuré.

Une autre possibilité est obtenue à l'aide d'une fibre en forme de $\mathrm{D}$ avec une biréfringence due à un coeur elliptique. Le mode supprimé est le mode $\mathrm{HE}_{11}$ pair avec un champ électrique dirigé suivant le petit axe du guide elliptique et normal à la partie plate de la fibre en $\mathrm{D}$ (Figure 16c).

Une configuration très simple utilise la biréfringence due à une courbure de la fibre. Si $n_{e}$ et $n_{0}$ sont respectivement les indices extraordinaires et ordinaires induits on $\mathrm{\delta} n=n_{e}-n_{0}=-0,133(a / R)^{2}$ où a est le rayon de la fibre et $R$ le rayon de courbure. On peut réaliser un contrôle de polarisation simplement en formant avec une fibre non biréfringente un nombre $\mathrm{N}$ de tours, de rayon $\mathrm{R}$ choisi de manière à obtenir un écart de phase de $\pi, \pi / 2$ entre les modes (Figure 17). La boucle est fixée en A et B. Quand elle est tournée d'un angle $\alpha$, le "twist" entre $\mathrm{B}$ et $\mathrm{C}$ tourne la polarisation incidente d'un angle proportionnel à $\alpha$. La même chose a lieu pour le "twist" inverse entre A et $\mathrm{C}$. Le composant est constitué de 3 enroulements dont les axes sont perpendiculaires à l'axe de la fibre et peuvent tourner de manière indépendante. Le premier et le dernier peuvent induire un déphasage de $90^{\circ}$ et celui du centre de $180^{\circ}$ (boucles de Lefebvre).

\section{7 - COMMUTATEUR}

Un commutateur optique est un composant qui applique ou non la puissance dans un guide d'onde. Une définition plus générale est celle d'un composant qui fait transiter la lumière d'un guide vers n'importe lequel d'un ensemble de guides adjacents. Un signal optique peut être commuté d'un canal à un autre de trois manières. Dans un premier cas un miroir, un prisme ou un autre système mécanique peut être physiquement placé dans le trajet d'un faisceau à dévier, par exemple entre deux fibres. La présence de parties mobiles implique une 

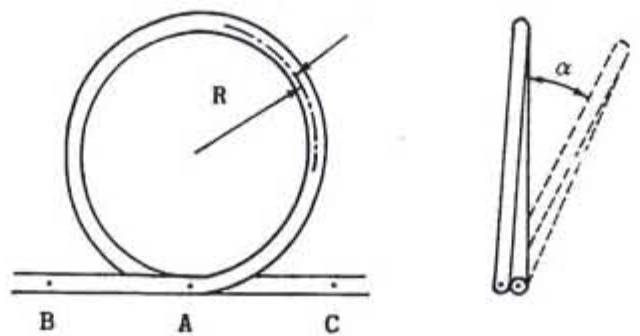

Figure 17

commutation lente et ces dispositifs sont utilisés dans des capteurs et non en télécommunications. Une seconde méthode donne une vitesse de commutation plus rapide, le signal optique est converti en signal électrique et c'est ce demier qui est commuté. La troisième méthode utilise la déflection de la puissance optique elle-même.

On peut utiliser un guide d'onde à fort indice placé entre deux demi blocs d'un coupleur à fibre polie. L'indice du guide est plus élevé que l'indice effectif du mode des fibres. Le rayonnement incident est couplé dans la seconde fibre (transmission croisée) ou recouplé dans le première fibre (transmission directe). La commutation est due à une variation de l'épaisseur du guide due par exemple à un élément piezoélectrique ou à une variation d'indice en utilisant une couche de cristal liquide. Les surfaces sont polies et la gaine est usée jusqu'à quelques microns du coeur. La longueur d'interaction est de l'ordre de $400 \mathrm{à} 500 \mu \mathrm{m}$, les blocs sont séparés de 10 à $20 \mu \mathrm{m}$. Les pics de puissance transmise sont $1.24,1.37 \mathrm{et}$ $1.53 \mu \mathrm{m}$. L'explication réside dans le battement de deux modes de symétrie, comme dans le cas du coupleur fondu étiré. La longueur de couplage est donné par $\pi / \Delta \beta$ où $\Delta \beta$ est la différence entre les constantes de propagation.

Un appareillage acousto-optique peut permettre de faire varier le taux de lumière électroniquement. Une rangée de transducteurs acoustiques est réalisée directement sur une fibre. La condition de Bragg qui permet à la lumière d'être éjectée de la fibre par interaction acousto-optique est $\sin \alpha=\mathrm{k}_{\mathrm{a}} / 2 \mathrm{k}$ où $\mathrm{k}_{\mathrm{a}}$ est le vecteur d'onde acoustique, $\alpha$ l'angle entre les vecteurs ka et $k$. La série de transducteur en phase avec une période $d$, existe les ondes longitudinales, avec un angle $\pm \beta$ par rapport à la normale tel que $\sin \beta=$ $2 \pi d / k_{\mathrm{a}}$. Si f est la fréquence centrale et $\mathrm{v}$ la vitesse acoustique, on obtient en égalant $\alpha$ et $\beta, f=v \sqrt{k} / \pi \mathrm{d}$. Les coupleurs non linéaires peuvent occuper une place importante dans les applications de commutation. La relation de non linéarité entre la polarisation électrique $\mathrm{P}$ et le champ électrique $\mathrm{E}$ dans une fibre optique conduit à une non linéarité de l'indice de réfraction. La susceptibilité du troisième ordre $\chi\left({ }^{(3)}\right.$ est responsable de l'effet Kerr optique. La susceptibilité du second ordre $\chi(2)$ n'intervient pas en principe dans les fibres à cause de l'inversion de symétrie de la silice fondue. L'effet Kerr conduit à l'auto modulation de phase d'impulsions lumineuses de forte puissance, se propageant le long de la fibre. Si le faisceau a une intensité I et si $n_{0}$ l'indice linéaire, on a: $\quad n(I)=n_{0}+n_{2} \quad I=n_{0}+n_{2} E^{2}(t)$ 
Une impulsion avec une enveloppe d'intensité $E^{2}(t)$ va induire une variation d'indice non linéaire $\Delta \mathrm{n}(\mathrm{t})=\mathrm{n}_{2} \mathrm{E}_{2}(\mathrm{t})$. Par suite, l'automodulation de phase $\Phi(\mathrm{t})$ du paquet d'onde est donnée par

$$
\Delta \Phi(t)=(2 \pi L / \lambda) \Delta n(t)=\left(2 \pi L n_{2} / \lambda\right) E^{2}(t)
$$

si $L$ est la longueur de fibre parcourue.

Si elle n'est pas contrôlée l'automodulation de phase et ses interactions avec les autres non linéarités (amplification Raman stimulée, diffusion Brillouin) induisent des dégradations dans les communications optiques. Par contre elle permet un certain nombre d'applications : solitons, compression d'impulsions.

Une commutation tout optique est possible basée sur une non linéarité. Dans des impulsions picosecondes un régime de dispersion normale, la puissance induit la commutation entre deux modes couplés linéairement, polarisées circulairement tournant en sens contraire, par biréfringence. De la même manière le recouvrement des champs évanescents couple les deux modes linéaires d'une fibre à deux coeurs.

\section{8 - MULTIPLEXEURS ET DEMULTIPLEXEURS}

Le concept de base du multiplexage est la transmission simultanée de l'émission modulée de plusieurs sources opérant avec différentes longueurs d'onde dans un même conducteur optique. Un exemple de communication avec trois canaux est montré figure 18. Pour la superposition et la séparation de plusieurs longueurs d'onde au commencement et à l'extrémité de la liaison, on utilise un multiplexeur et un démultiplexeur. Trois diodes (diodes lasers ou diodes électroluminescentes) $\mathrm{L}_{1}, \mathrm{~L}_{2}, \mathrm{~L}_{3}$ envoient la lumière dans le multiplexeur et ensuite dans la fibre. Trois PIN ou photodiodes à avalanche recoivent le signal à travers le démultiplexeur. Dans le cas de la figure 19a, la ligne est unidirectionnelle; dans le cas de la figure $19 \mathrm{~b}$, à chaque extrémité, on place un multiplexeur - démultiplexeur.

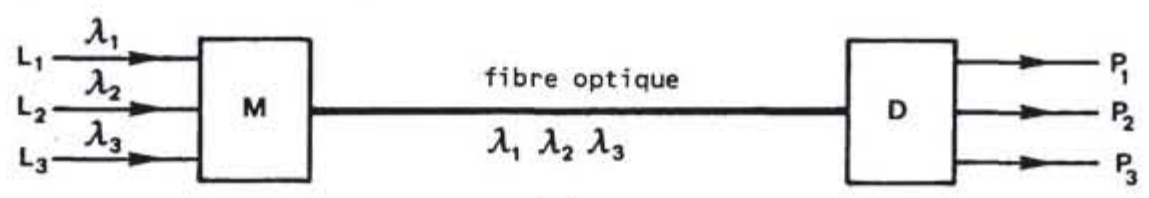

(a)

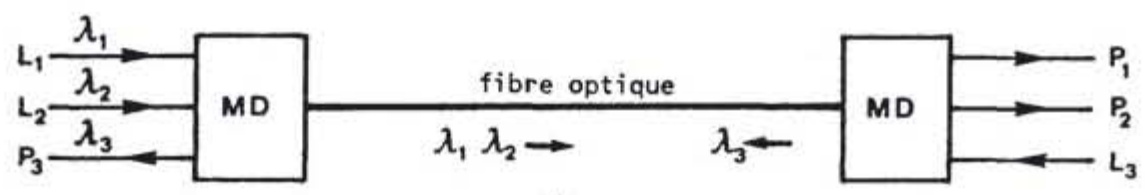

(b)

Figure 18 
Les principes physiques utilisés dans ces composants sont nombreux : images formées par des lentilles ou des miroirs concaves, dispersion à partir d'interférences dues à des structures multicouches ou à base de diffraction par des réseaux (Figure 14). Une méthode utilise une fibre monomode collée sur une rainure convexe usinée sur un bloc de silice. Après polissage sur la surface, un réseau de période $S$ est réalisé. Une réflectivité de $98 \%$ dans le premier ordre pour une longueur d'onde et une largueur de raie de $0,8 \mathrm{~nm}$ ont été obtenus. Les applications concernent aussi la sélection d'un mode laser, la commutation et la modulation non linéaire.

\section{9 - REFLECTEURS}

On réalise un réflecteur en formant une boucle de fibre entre les deux bords d'un coupleur à fibre à faible perte (Figure 19). Celui-ci est fabriqué avec la technologie de fusion étirage à partir d'une simple longueur de fibre. On réduit ainsi les réflections parasites et les pertes additionnelles dues aux épissures. La réflectivité dépend des caractéristiques spectrales du couplage et du degré de biréfringence de la boucle.

Ils sont utilisés comme composants passifs dans les Fabry-Perot et pour former des cavités résonantes dans les lasers à fibres. Ils sont aussi utilisés comme composants pour faire varier la longueur d'onde et dans les mémoires à ligne à retard.

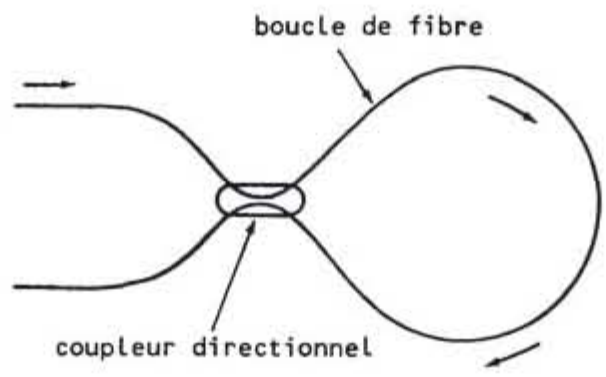

Figure 19

\section{0 - LASERS ET AMPLIFICATEURS}

Des sources lasers à fibre à bas seuil utilisant des résonateurs en anneau ont été mises au point incluant les lasers Brillouin, Raman réalise dans les versions à fibres monomodes en utilisant des coupleurs à champ évanescent.

La fibre optique dopée $\mathrm{Ge} 02$ a été étudiée comme milieu d'optique non linéaire du fait que la section efficace de diffusion de ce verre semble d'un ordre de grandeur plus grand que le verre de silice. Avec une fibre optique monomode dopée $\mathrm{Ge}_{2}$ de diamètre de coeur $2 \mu \mathrm{m}$ et des pertes inférieures à $20 \mathrm{~dB}$ $\mathrm{km}^{-1}$, seulement $1 \mathrm{~W}$ de puissance de pompe est nécessaire pour la génération d'effet Raman. Cette puissance est facile à obtenir avec une diode laser pulsće. 
Les lasers et amplificateurs à fibres monomodes dopées avec des terres rares devraient trouver des applications importantes des télécommunications et dans le domaine des capteurs. Par exemple la largeur de la bande de fluorescence d'ions terre rare dans la silice peut permettre la réalisation de sources accordables et d'amplificateurs. Des réflecteurs en anneaux sont utilisés dans le cas de lasers à fibres dopées $\mathrm{Nd}^{3+}$ dans la transition ${ }^{4} \mathrm{~F}_{1 / 2} \rightarrow{ }^{4} \mathrm{I}_{11 / 2}$. Par exemple un laser à $\mathrm{Nd}^{3+}$ accordable, qui opère de manière continue à température ambiante, a été réalisé couvrant un domaine s'étendant de 0,9 à 0,945 et de 1,070 à $1,135 \mu \mathrm{m}$. Une puissance de sortie de $3 \mathrm{~mW}$ est obtenu pour $53 \mathrm{~mW}$ de pompe pour les deux domaines en pompant avec un laser à colorant à $590 \mathrm{~nm}$ (Figure 20a).

Les lasers à fibre dopée $\mathrm{Er}^{3+}$ opèrent à $1,55 \mu \mathrm{m}$ et sont très étudiés en vue des télécommunications. La réflection peut être obtenue à l'aide d'un réseau (Figure 20b).

Un décalage constant dans la réflectivité du coupleur de sortie de la cavité en fonction de la longueur d'onde peut être induite par effet thermo-optique. Celui ci change la dépendance spectrale du rapport de couplage d'un coupleur à fibre fusionné dans un réflecteur en anneau. Cette réflectivité variable a deux intérêts : le premier est l'accordabilité en longueur d'onde et le second est l'utilisation du réflecteur en anneau variable comme coupleur de sortie ajustable (Figure 20c).
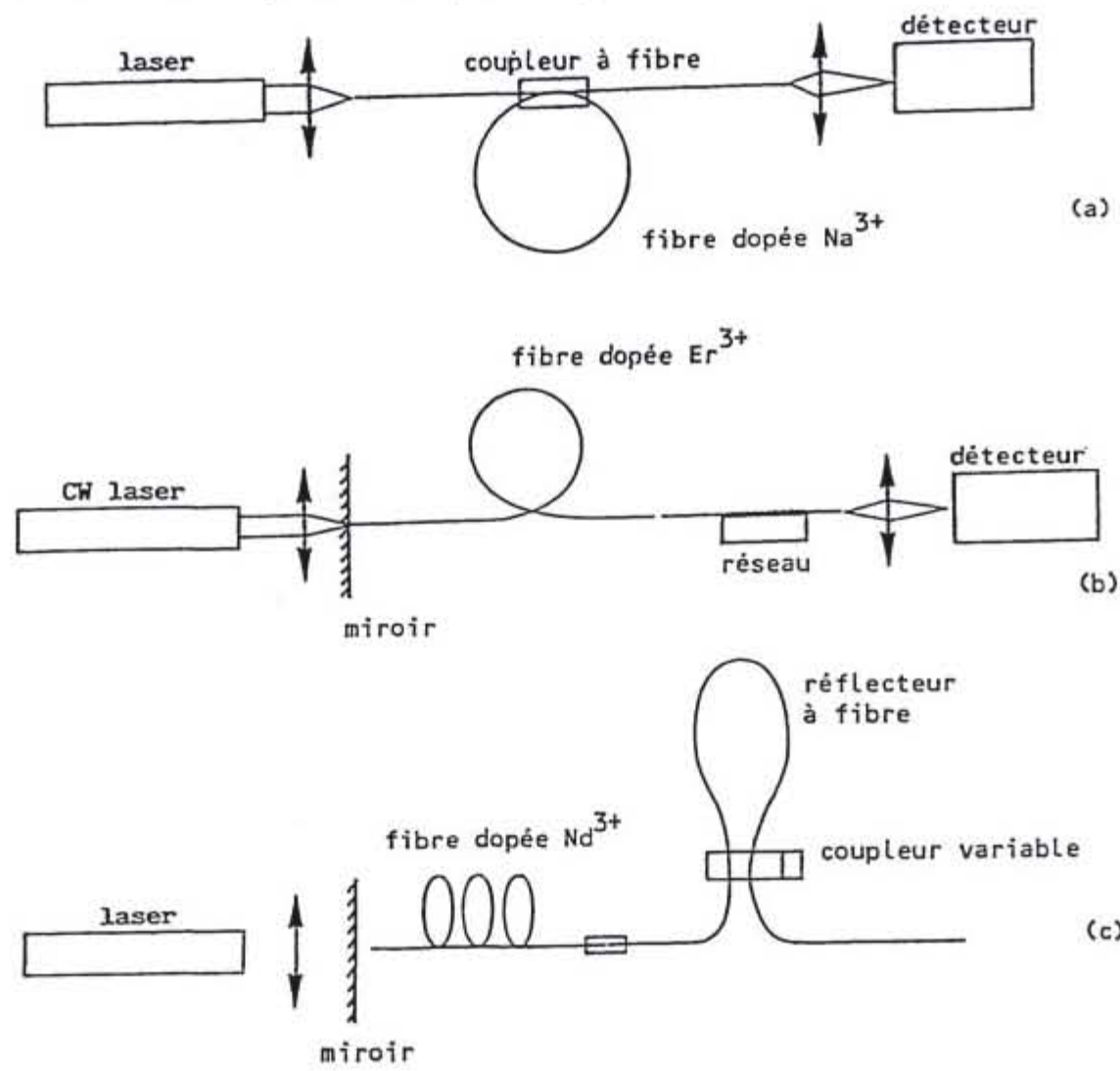

Figure 20 
Pour faire laser à une longueur d'onde donnée on introduit une rétroaction optique en utilisant un coupleur faible perte et un réseau blasé en réflection. Le réflecteur en anneau réglable agit alors comme un réflecteur de sortie variable en transmission. Pour une puissance de pompe constante, la puissance de sortie peut varier de manière continue.

\section{1 - MODULATEURS}

Un modulateur agit sur la phase de l'onde optique quand une contrainte est appliquée sur une fibre monomode, la phase de la lumière change. Un modulateur simple est constitué d'une fibre optique gainée, entourée d'un transducteur piezoélectrique cylindrique et creux. Il est utilisé dans les interféromètres pour corriger le glissement de phase.

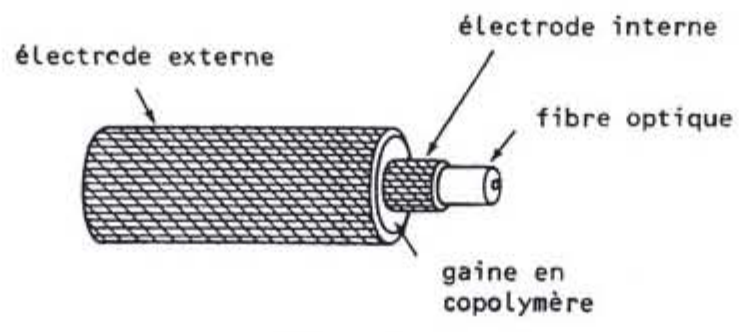

Figure 21

Une méthode de fabrication consiste à enrober la fibre dans un film plastique piezoélectrique. La contrainte appliquée sur le film plastique à l'aide d'un champ électrique est transmise à la fibre. Des fibres revêtues avec une gaine en polyvinylidène (PVD) ou en co-polymer à base de fluoride de polyvinylidène (PVDF) ont été fabriquées. La fibre gainée de plastique avec des électrodes coaxiales a la géométrie d'un long et fin cylindre (Figure 21). La phase de la lumière se propageant dans une fibre à saut d'indice d'une longueur $L$ est définie par :

$$
\varphi=B \mathrm{~L}=\mathrm{knL}
$$

et quant la fibre est soumise aux effets de modulations un changement de phase $\Delta \varphi$ est introduit :

$$
\Delta \varphi=B \Delta L+L \Delta B=k n L S_{z}+k l \Delta n
$$

où $\mathrm{Sz}$ est la contrainte axiale et représente le changement de longueur ; le second terme est celui du changement de l'indice de réfraction dù à l'effet photoélastique. Si le champ de pression induit par la contrainte radiale est uniforme, on a :

$$
\Delta \varphi=k n L\left(S_{z} \frac{n^{2}}{2}\left[\left(P_{11}+P_{11}\right) S_{t}+P_{12} S_{z}\right]\right)
$$

ou les Pij sont les coefficients photoélastiques. 
La réponse optique du changement de phase induite par les tensions continues appliquées a pour valeur expérimentale $0,8010^{-5} \mathrm{rad} \mathrm{V}^{-1}$ par mètre de fibre sur une large plage de basses fréquences $(20 \mathrm{~Hz}-20$ H. La modulation de phase a été mise en évidence sur une large plage de fréquence $20-50 \mathrm{MHz}$ avec une réduction en phase de $3,510^{-6} \mathrm{rad} \mathrm{V}^{-1} \mathrm{~m}$ pour une gaine plastique de $50 \mathrm{à} 100 \mu \mathrm{m}$. Un autre type de modulateur utilise l'effet Kerr optique qui induit un changement d'indice de réfraction par propagation d'une forte intensité optique.

\section{2 - INTERFEROMETRES}

La variation de phase d'une onde optique due à des influences extérieures est utilisée pour réaliser des capteurs. Le système le plus connu est l'interféromètre de Mac Zenhder dont le principe est rappelé figure 22. Si l'on utilise des fibres monomodes comme bras de l'interféromètre les deux miroirs deviennent inutiles et les séparateurs sont remplacés par des coupleurs.

\section{3 - CONCLUSION}

Les plus importants composants à fibre ont été très succinctement présentés dans cet article. Il peuvent aussi se combiner avec des circuits en optique intégrée ce qui multiplie les possibilités. Toutefois, à l'heure actuelle, une grande partie reste encore dans le domaine de la Recherche et du Développement sauf en ce qui concerne les coupleurs et les amplificateurs. D'autres applications peuvent être signalées par exemple en traitement du signal.

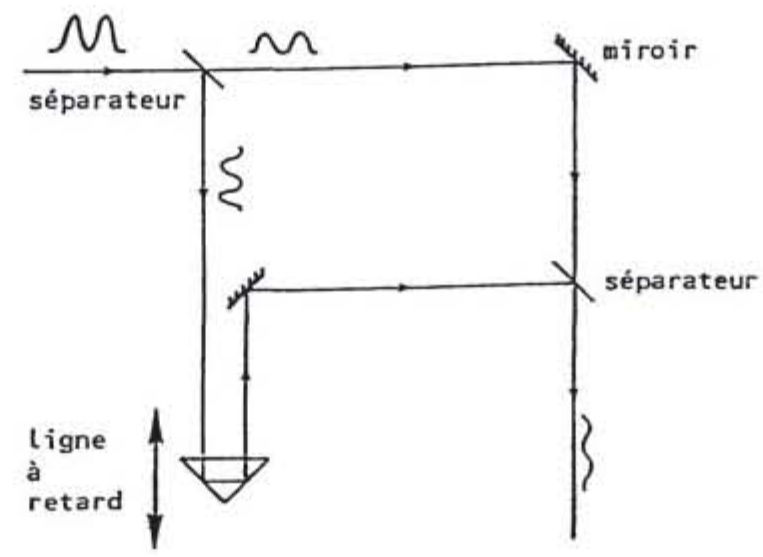

Figure 22 
Tableau I

Composants à fibre linéaires et non linéaires

\begin{tabular}{|c|c|c|c|}
\hline COMPOSANT A FIBRE & TYPE & PRINCIPE PHYSIQUE & FABRICATION \\
\hline Expanseur de faisceau & MM/SM & Croissance du diamètre du faisceau & Fibre étirf́e avec microlentille \\
\hline Coupleur directionnel & & Onde évanescente & $\begin{array}{l}\text { Fibre polie avec liquide adaptateur } \\
\text { d'indice }\end{array}$ \\
\hline $\begin{array}{l}\text { Indépendant de la polarisation } \\
\mathrm{X}, \mathrm{Y} \text { coupleurs } \\
\text { nxn couleurs }\end{array}$ & $\begin{array}{l}\text { MM/SM } \\
\text { MM/SM }\end{array}$ & $\begin{array}{l}\text { Couplage des ondes / ou } \\
\text { Couplage par micro optique }\end{array}$ & $\begin{array}{l}\text { Torsadées et fondus / ou } \\
\text { avec lentille SELFOG ou microprismes }\end{array}$ \\
\hline $\begin{array}{l}\text { Dépendant de la polarisation } \\
\mathrm{X} \text { à maintien } \\
\mathrm{X} \text { séparateur }\end{array}$ & $\begin{array}{l}\text { PMSM } \\
\text { PMSM }\end{array}$ & & \\
\hline Filtres de longueur d'onde & SM & $\begin{array}{l}\text { Perte par courbure / Couplage du } \\
\text { coeur a un guide de fort indice / } \\
\text { Couplage direct par accord de phase } \\
\text { / Sellection de longueur d'onde }\end{array}$ & $\begin{array}{l}\text { Fibre monomode polie avec liquide } \\
\text { adaptateur IDEM+supertrat en sitice } \\
\text { Coupleur coascial escpanseur } \\
\text { Reseau gravé sur fibre polie }\end{array}$ \\
\hline Décalage en longueur d'onde & $\begin{array}{l}\text { SM } \\
\text { PMSM }\end{array}$ & $\begin{array}{l}\text { Couplage par onde évanescente } \\
\text { Entre } \mathrm{LP}_{01} \text { et } \mathrm{LP}_{11} \\
\text { Couplage de deux polarisations } \\
\text { d'une fibre birefringente }\end{array}$ & $\begin{array}{l}\text { Micro courbures périodiques sur la fibre } \\
\text { par serrage ou onde acoustique } \\
\text { Contrainte spatiale périodique ou onde } \\
\text { acoustique }\end{array}$ \\
\hline
\end{tabular}




\begin{tabular}{|c|c|c|c|}
\hline Polariseur & PMSM & $\begin{array}{l}\text { Couplage évanescent d'un état de } \\
\text { polarisation } \\
\text { Effet de courbure (changement } \\
\text { d'indice) }\end{array}$ & $\begin{array}{l}\text { Utilisation d'un craistal bireffringent ou } \\
\text { d'un film métallique mince ou d'une } \\
\text { fibre attaquée chimiquement ou d'une } \\
\text { fibre en D métaliscé. } \\
\text { Spire de fibre ( } N / 4 \text { ou } \lambda / 2 \text { lame) ou fibre } \\
\text { birefringente }\end{array}$ \\
\hline Commutateurs & $\begin{array}{l}\mathrm{MM} \\
\mathrm{SM}\end{array}$ & $\begin{array}{l}\text { Couplage } \\
\text { Variation d'épaisseur par } \\
\text { piezoelectique ou variation d'indice } \\
\text { Diffraction de Bragg } \\
\text { Effet Kerr optique }\end{array}$ & $\begin{array}{l}\text { Transducteur acoustique sur la fibre } \\
\text { Fibre à deux coeurs }\end{array}$ \\
\hline Multi-demultiplesceurs & $\begin{array}{l}\mathrm{MM} \\
\mathrm{SM}\end{array}$ & $\begin{array}{l}\text { Combinaison de divers } \\
\text { cannaux de longueur d'onde }\end{array}$ & Coupleur directionnel ou filtre \\
\hline Reflecteurs & SM & Reflectivité sur une spire & Coupleur fusion étirage et spire \\
\hline Lasers et amplificateurs & PM & $\begin{array}{l}\text { Diffusion Brillouin Stimulée } \\
\text { Diffusion Raman } \\
\text { Spectre de fluorescence } \\
\text { Onde évanescente }\end{array}$ & $\begin{array}{l}\text { Fibre silice ou fibre dopées } \mathrm{Ge}^{\circ}, \mathrm{P}, \mathrm{H} 2 \\
\text { pour modifier le gain } \mathrm{Raman} \\
\text { Fibre monomode dopé terre rare } \\
\quad \mathrm{Nd}^{3+}, \mathrm{Er}^{3+} \\
\text { Solution de colorant sur un coeur de } \\
\text { fibre }\end{array}$ \\
\hline Interféromètres & $\begin{array}{l}\text { SM } \\
\text { PMSM }\end{array}$ & $\begin{array}{l}\text { Interférences entre } \\
\text { deux ondes }\end{array}$ & $\begin{array}{l}\text { Deux fibres monomodes dans les deux } \\
\text { bras ou un bras avec deux polarisations }\end{array}$ \\
\hline Modulateurs & SM & Effet photoélastique & Fibre entourée de copolymère \\
\hline
\end{tabular}




\section{Références}

[1] Stolen R H and De Paula R P. 1987 Proc. IEEE 75, 1498

[2] Goure J P, Verrier I, Meunier J P 1989 J. Phys. D. Applied Phys 22179

[3] Miller C M, Mettler S C and White I A 1986 Optical fiber splices and connectors - theory and methods (Marcel Dekker, New-York)

[4] Hunsperger R G 1985 Integrated optics: Theory and Technology 2nd edition (New-York, Springer Verlag)

[5] Neumann E G 1988 Single-mode fibers - Fundamentals chapter 12 (Springer Verlag)

[6] Taylor H F 1987 Proc. IEEE 751524

[7] Noda J and Yokohama I 1988 Fiber devices for fiber sensors Technical Digest 2468 (Washington D.C, O.S.A.)

[8] Dakin J P. and Culshaw B 1988 Optical fibre sensors 1 : Principles and components (London, Artech House)

[10] Jones B E (Ed) 1987 Current advances in Sensors (Bristol, Adam Hilger)

[11] Dakin J P. and Culshaw B 1989 Optical fibre sensors 2: Systems and applications (London, Artech House)

[12] Snyder A W and Love J D Optical Wave guide theory Chapsman and Hall

[13] Baker L.R 1988 Fibre optics 88 S.P.I.E. Proc. 949 (Bellingham, S.P.I.E.)

[14] Jeunhomme L B 1983 Single-mode fibre optics : Principles and applications (Marcel Dekker, New-York)

[15] Culshaw B 1984 Optical fibre sensing and signal processing Peter Peregrinus Ltd

[16] Agarwal A K 1985 Fiber and Integrated Optics 627

[17] Carenco A 1991 Ecole d'été Cargèse 1991 - cet ouvrage

[18] Valette S 1991 Ecole d'été 1991 - cet ouvrage 